\title{
Mood Mirroring with an Embodied Virtual Agent: A Pilot Study on the Relationship Between Personalized Visual Feedback and Adherence
}

\author{
Simon Provoost ${ }^{1,2(\bowtie)}$, Jeroen Ruwaard ${ }^{1,2,3}$, Koen Neijenhuijs ${ }^{1,2}$, \\ Tibor Bosse ${ }^{4}$, and Heleen Riper ${ }^{1,2,3,5}$ \\ ${ }^{1}$ Section of Clinical Psychology, Vrije Universiteit Amsterdam, \\ Amsterdam, Netherlands \\ \{s.j.provoost, j. j. ruwaard,k.i.neijenhuijs, \\ h.riper\}@vu.nl \\ 2 Amsterdam Public Health, Amsterdam, Netherlands \\ 3 GGZ inGeest, Amsterdam, Netherlands \\ ${ }^{4}$ Department of Computer Science, Vrije Universiteit Amsterdam, \\ Amsterdam, Netherlands \\ t.bosse@vu.nl \\ 5 University of Southern Denmark, Odense, Denmark
}

\begin{abstract}
Human support is thought to increase adherence to internet-based interventions for common mental health disorders, but can be costly and reduce treatment accessibility. Embodied virtual agents may be used to deliver automated support, but while many solutions have been shown to be feasible, there is still little controlled research that empirically validates their clinical effectiveness in this context. This study uses a controlled and randomized paradigm to investigate whether feedback from an embodied virtual agent can increase adherence. In a three-week ecological momentary assessment smartphone study, 68 participants were asked to report their mood three times a day. An embodied virtual agent could mirror participant-reported mood states when thanking them for their answers. A two-stage randomization into a text and personalized visual feedback group, versus a text-only control group, was applied to control for individual differences (study onset) and feedback history (after two weeks). Results indicate that while personalized visual feedback did not increase adherence, it did manage to keep adherence constant over a three-week period, whereas fluctuations in adherence could be observed in the text-only control group. Although this was a pilot study, and its results should be interpreted with some caution, this paper shows how virtual agent feedback may have a stabilizing effect on adherence, how controlled experiments on the relationship between virtual agent support and clinically relevant measures such as adherence can be conducted, and how results may be analyzed.
\end{abstract}

Keywords: Ecological momentary assessment $\cdot$ Virtual agent Feedback · Adherence 


\section{Introduction}

Internet-based psychotherapeutic interventions, also referred to as eMental Health interventions, can be effective in the treatment of various mental disorders when compared to face-to-face interventions [1]. Many interventions that target common mental health disorders such as mood, anxiety, and substance use disorders, are based on cognitive behavioral therapy (CBT). Internet-based CBT interventions are either guided, or self-guided, with guidance usually being provided by health professionals or trained volunteers. It has been found that guided interventions are generally more clinically effective, e.g. reductions in symptomatology, than unguided interventions [2]. While the precise contribution of human support remains unclear, a number of working mechanisms have been suggested [3]. One such mechanism is that human support contributes to patients' motivation to complete an intervention, which in turn may increase adherence [4]. Indeed, it has been shown that adherence may be superior when human support is available [5], and that non-optimal exposure or non-adherence to interventions, e.g. not completing exercises or dropping out of interventions early, tends to reduce their clinical effectiveness [6].

The study described in this article is part of a project in which we are looking to bridge the gap between guided and unguided internet-based CBT interventions by automating support through the use of embodied virtual agents. From a literature review of their application in the treatment of common mental health disorders, we concluded that few studies have explored their use in a supportive role to online CBTbased interventions. Although a number of applications seemed feasible and promising, there is still little evidence for their impact on clinically relevant outcomes such as symptom reduction or adherence [7]. Clinical psychology is an applied science, however, which means that there is a strong emphasis on empirical validation when introducing novel technologies. Although from a technological perspective, lots of interesting solutions have been, and are being developed, they cannot be applied in clinical practice without such validation. The present study represents the first in a series of controlled studies in which we aim to discover how and whether virtual agent support can contribute to eMental Health interventions' clinical effectiveness.

Because a detailed study of clinical outcomes in controlled settings, such as symptom severity, requires clinical study populations (ethical implications) and followup measurements (long timespan), we chose to study adherence as an outcome measure in this pilot study, on the assumption that it is a potential mediator for clinical effectiveness. We opted for Ecological Momentary Assessment (EMA) as an intervention strategy, also referred to as experience sampling, which refers to the repeated sampling of subjects' current behaviors and experiences in real time, in subjects' natural environments [8]. EMA can be a component of internet-based CBT interventions, for example to measure fluctuations in mood [9]. Additionally, it is a clear measure of adherence, as patients either do or do not respond to EMA requests.

In the remainder of this paper we describe our explorative pilot study, in which we test the hypothesis that virtual agent support can increase adherence to EMA requests. With respect to the virtual agent design, we opted for a simple approach that fulfills the ECA criteria (the agent has an embodiment, communicates with the user, and uses a 
form of reasoning to simulate agency [10]) in a minimalistic manner. Although frameworks exist for the development of ECAs with their full range of verbal and nonverbal capabilities (e.g. [11, 12]), using them for the development of virtual agents, and their subsequent integration with existing EMA platforms, was considered too timeconsuming for this study. Moreover, agents do not necessarily need to be very complicated for motivational purposes, because it has been shown that even the mere presence of an embodied agent can improve user motivation, for example when shown next to a chat dialog box in which instructions and feedback for an interactive game are displayed [13]. A more detailed description of the experiment and the agent's design is provided in the Methods section.

\section{Methods}

\subsection{Design}

We conducted an explorative controlled pilot study with a two-stage randomized between-subject design. Participants self-monitored their mood on a smartphone EMA application in which a virtual character could give personalized visual feedback to user responses by mirroring their reported mood state. Approval for the study was obtained from the Research Ethics Committee of the Faculty of Movement and Behavioural Sciences of the Vrije Universiteit Amsterdam (reference number: VCWE-2016-014).

Before study onset, participants were randomly allocated to either a text + personalized visual feedback or a text-only feedback condition, to control for individual differences in initial motivation and other potentially relevant background variables. A known issue with prolonged interaction with virtual agents is that it may become repetitive, leading to a decline in motivation and willingness to interact with a system [14]. Because our agent's design is fairly simplistic we wanted to control for this effect, and therefore, two weeks into the study, participants were randomized again to control for feedback history. Because no changes to the application could be made while the study was ongoing, randomization for the entire study took place before study onset. Participants were assigned to one of four possible groups, each with a different combination of text + personalized visual feedback $(\mathrm{F})$ or text-only feedback $(\mathrm{N})$ during weeks 1-2 and week 3 .

\subsection{Procedure}

Before the study started, participants received an email with an invitation to fill out a digital informed consent form and demographic questionnaire. After giving their consent, participants installed the Android-only EMA application on their mobile devices. The application automatically stopped sending EMA requests once the study was over, after which it could be removed from the participants' smartphones. 


\subsection{Participants}

As part of a research project for a bachelor's degree, students were asked to recruit at least 10 adults from their social network. Inclusion criteria for participation were (1) age 18 years or older, (2) owning an Android smartphone (minimal Android 2.3), and (3) no known severe mental health problems. Participants did not receive financial compensation, and were told that their participation would benefit the education of the student who had approached them.

\subsection{Materials}

Ecological Momentary Assessment of Mood. To collect self-monitored mood data, we built an Android smartphone application using the movisensXS EMA framework [15]. The app prompted participants to rate their mood on their smartphone at three set time points each day (11:00, 15:00, and 20:00). Mood was assessed through the circumplex model of affect [16], which conceptualizes affective states as two-dimensional constructs comprising different levels of valence and arousal. Previous studies measured valence and arousal through 5-point scales [17]. We decided to tap both dimensions on a 3 -point scale scored from -1 to 1 (negative to positive; low to high) (Fig. 1), as this allowed a direct mapping to the visual feedback presented in the next section (Fig. 2).
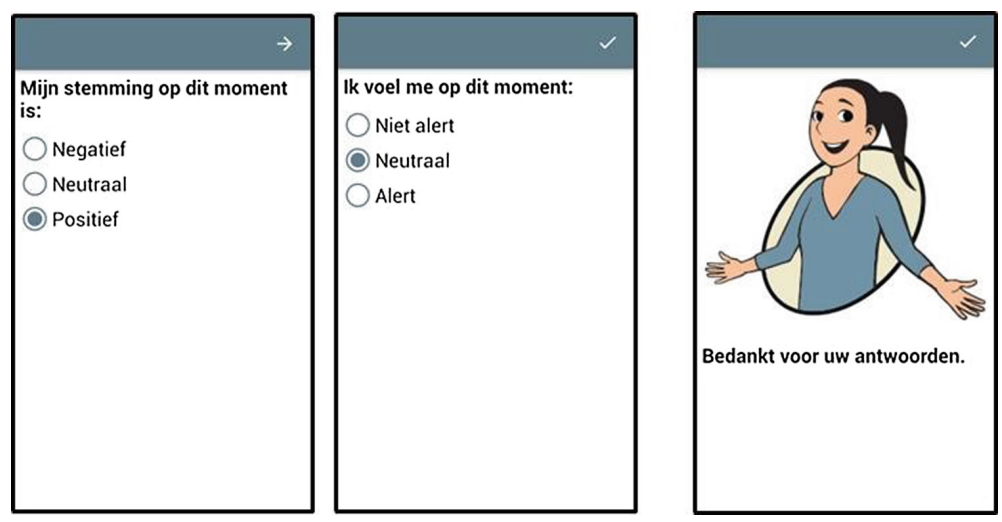

Fig. 1. Screenshots of an EMA response and the system's reply including personalized visual feedback (translated from Dutch): left: "My mood at this moment is [Negative; Neutral; Positive]", middle: "At this moment I feel [Not alert; Neutral; Alert]", right: "Thank you for your answers"

Personalized Visual Feedback With a Virtual Agent. After responding, participants received a message on a third screen, thanking them for their answers. In the personalized visual feedback condition, an embodied virtual agent accompanied this message. It consisted of the female version of the Pick-A-Mood (PAM) model [18] that matched the reported mood. For example, a participant reporting positive valence $(+1)$, 
and high arousal (+1), was deduced to be in an excited mood (Fig. 2). Our visual feedback can be considered a simple form of empathy, where the system deduces and reflects users' moods based on their response to the EMA requests. With this feedback, we hoped to operationalize two concepts of Dialogue Support from Persuasive System Design [19], the inclusion of which has been found to increase adherence [20]: social role ("if a system adopts a social role, users will more likely use it for persuasive purposes") by accompanying the thank you message with the face of a virtual character, and similarity ("people are more readily persuaded through systems that remind them of themselves in some meaningful way") by having the character mirror the participant-reported mood.

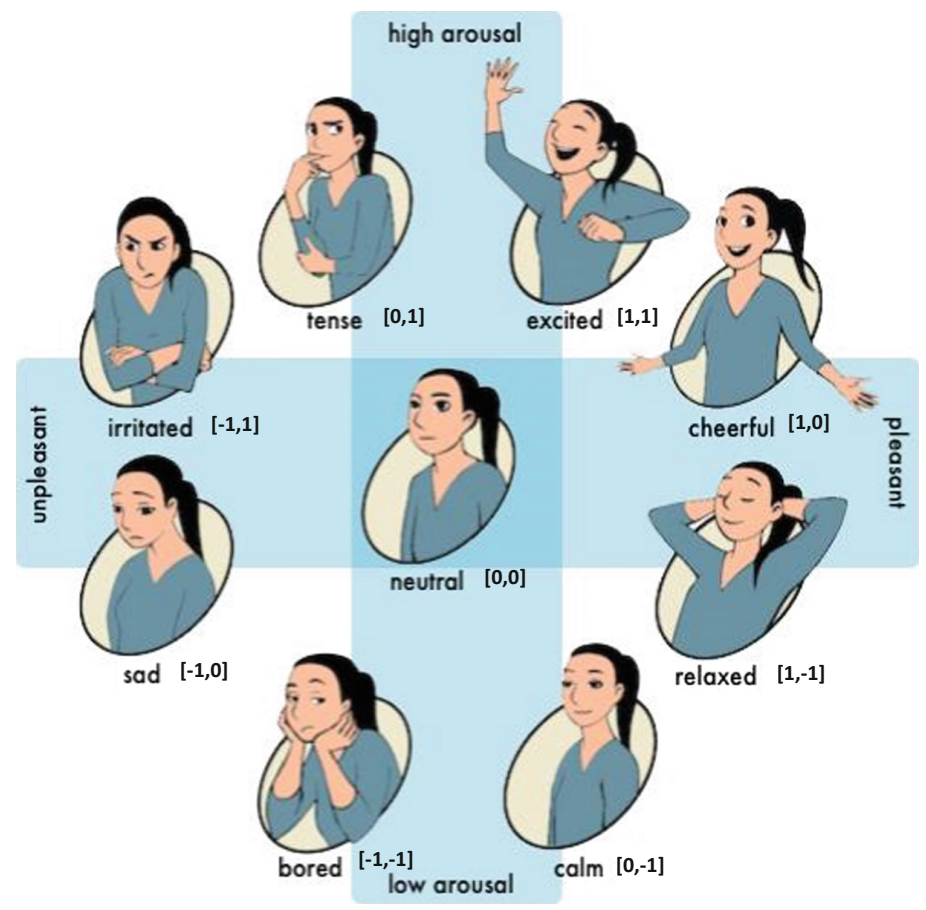

Fig. 2. The Pick-A-Mood model, including an interpretation of mood ratings [Valence, Arousal]. Note that the model had to be tilted slightly clockwise to allow for the interpretation.

\subsection{Measures}

Adherence. Adherence was represented as a vector of binary values indicating either a response $(=1)$ or no response $(=0)$ to EMA requests on subsequent trials.

Feedback. Feedback was represented as a vector of binary values indicating either text + personalized visual feedback $(=1)$, or text-only feedback $(=0)$, accompanying the 'thank you for your answers' message. 
Time. All trials received a sequentially ordered 'time stamp' ranging from the first to the last trial (Range $=1-63$ ). Trials that were missing due to technical issues were added to the dataset, but with $N A$ values for adherence, such that all 63 trials were accounted for.

\subsection{Statistical Analysis}

In our model, we assumed effects of time (people naturally lose interest in EMA after a while [21]), feedback, and individual differences (some people may be more adherent to start with). To analyze the relationship between time and feedback as independent variables, and adherence as dependent variable, we used the glmer function from the lme4 statistical package [22] in the R-environment (version 3.3.2) [23]. For our main hypothesis, we conducted a logistic mixed effects analysis, with a 'feedback (1/0) $\mathrm{x}$ time (1-63)' interaction as fixed effect, and adherence (1/0) as the dependent variable. To investigate complex patterns over time, contrasts for the time variable were set to polynomials up to the tenth power. We accounted for individual differences at onset by adding random intercepts for the different participant IDs to our model. The regression model looked as follows in R-syntax:

$$
\operatorname{adh} \sim \text { feedback } * \text { time }+(1 \mid \text { id })
$$

\section{Results}

\subsection{Participant Flow}

A total of 85 participants were recruited and randomized to one of the four groups, with 17 dropping out entirely or failing to install the software on time. From the 68 participants who had started, another 7 dropped out, and 7 were excluded from the analysis as they had experienced technical issues that resulted in either too few EMA requests (e.g. 0 to 2 per day on many different days), or too many (e.g. 4 per day) having been logged in the movisensXS data export files. Our final dataset included 54 participants (see Fig. 3).

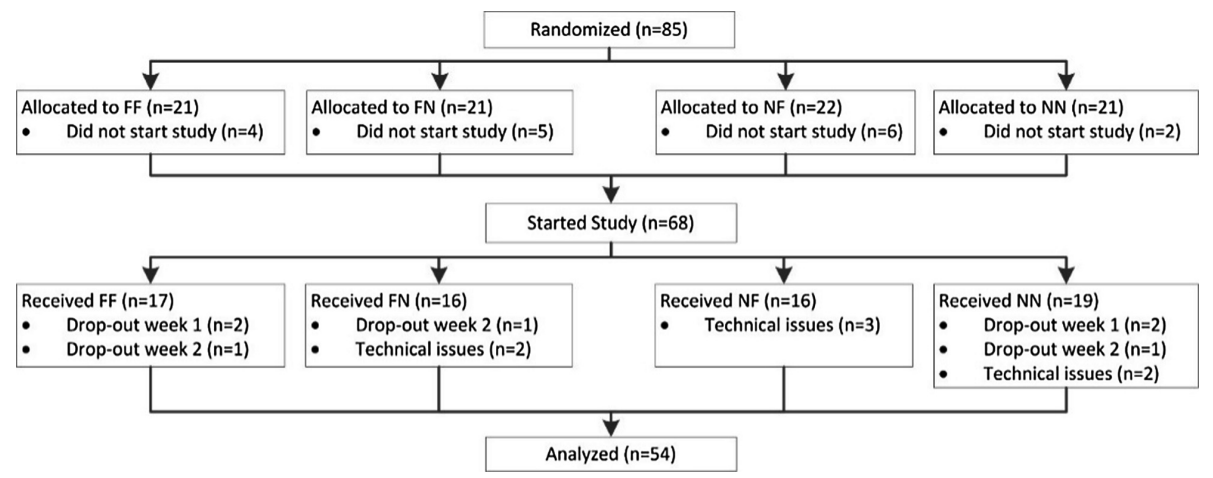

Fig. 3. Participant flow 


\subsection{Descriptive Statistics}

Participants. 49 participants, 24 males and 25 females with mean age of 29.31 (Range $=20-64)$ filled out the demographic questionnaire, while 5 participants failed to do so.

Adherence. The 54 participants in the final dataset responded to EMA requests in 2004 out of a total of 3080 trials $(M=37.1$ responses, and $M=57.0$ trials per participant), resulting in an overall adherence of $65.1 \%$. Note that the total number of trials does not add up to 63 on average ( 3 measurements per day for 21 days), as some requests were missed, either on the first day (e.g. when a participant started with the 20:00 measurement), or due to technical issues that prevented trials from taking place.

Feedback. The personalized visual feedback group received 1497 (48.6\%) requests, and responded to $971(64.9 \%)$. The text-only group received $1583(51.3 \%)$ requests, and responded to $1033(65.3 \%)$. The valence question was answered slightly more positively in the text-only condition, but no large differences existed between the two groups. With respect to the visual feedback provided, excited $(\mathrm{n}=317(32.7 \%))$ and neutral $(\mathrm{n}=241,(24.8 \%))$ were most prominent.

Time. A total of 322 trials were unaccounted for due to technical issues, 183 of which would have contained personalized visual feedback. For our analysis, these trials were added to our dataset with missing values for adherence, giving us a total of 3402 trials. With the ten polynomial contrasts for the time variable our model was able to run, while visual inspection showed that no more than ten polynomials were to be expected.

\subsection{The Effect of Feedback}

Mixed Effects Logistic Analysis. Summary results of the mixed effects logistic analysis, based on all 3402 trials, are depicted in Table 1 below. Significant effects were found for feedback $(p=.03)$, as well as the feedback $*$ time interaction for the $3^{\text {rd }}$ $(p=.01), 7^{\text {th }}(p=.01)$, and $9^{\text {th }}(p<.01)$ order.

Feedback * Time Interaction. Because of the significant interaction effects, the main effect of feedback cannot be interpreted as such. To further disentangle the model, the analysis was conducted again for both the feedback and no feedback condition, consequently leaving the feedback variable out of the equation. The results of these analyses with regard to the previously significant interaction effects are depicted in Table 2 below.

Whereas no significant effects of time were found for the feedback condition, the significant $3^{\text {rd }}(p=.04), 7^{\text {th }}(p=.02)$, and $9^{\text {th }}(p<.01)$ order effects of time remained in the text-only feedback condition. A Chi-square test of the entire model further confirmed the significant feedback * time interaction effect $\left(X^{2}(62)=84.99, p=.03\right)$. To visualize this, Fig. 4 illustrates fluctuations in the no feedback condition (top left), which can be disseminated into separate polynomials of the $3^{\text {rd }}$ (two bends; top right), $7^{\text {th }}$ ( 6 bends; bottom left), and $9^{\text {th }}$ ( 8 bends; bottom right) power. Meanwhile, the feedback condition is stable across all trials. 
Table 1. Summary of the logistic mixed effects analysis results

\begin{tabular}{|c|c|c|c|c|}
\hline Variable & Estimate & Standard error & z-value & P-value \\
\hline Feedback & 0.13 & 0.06 & 2.19 & $0.03 *$ \\
\hline Time & 0.09 & 0.35 & 0.27 & 0.79 \\
\hline Feedback * time & 0.07 & 0.42 & 0.17 & 0.86 \\
\hline Feedback $*$ time $^{2}$ & 0.56 & 0.37 & 1.49 & 0.14 \\
\hline Feedback $*$ time $^{3}$ & 0.93 & 0.37 & 2.53 & $0.01 *$ \\
\hline Feedback $*$ time $^{4}$ & 0.50 & 0.37 & 1.35 & 0.18 \\
\hline Feedback $*$ time $^{5}$ & -0.42 & 0.36 & -1.18 & 0.24 \\
\hline Feedback $*$ time $^{6}$ & 0.42 & 0.35 & 1.18 & 0.24 \\
\hline Feedback $*$ time $^{7}$ & 0.91 & 0.35 & 2.60 & $0.01 *$ \\
\hline Feedback $*$ time $^{8}$ & -0.08 & 0.35 & -0.23 & 0.82 \\
\hline Feedback $*$ time $^{9}$ & -1.21 & 0.35 & -3.45 & $0.00 * *$ \\
\hline Feedback $*$ time $^{10}$ & -0.01 & 0.35 & -0.02 & 0.98 \\
\hline
\end{tabular}

Table 2. Disentanglement of the significant interaction effects from the main analysis

\begin{tabular}{l|l|r|l|l|l}
\hline & Variable & Estimate & Standard error & z-value & P-value \\
\hline Visual feedback & time $^{3}$ & -0.65 & 0.52 & -1.26 & .21 \\
\cline { 2 - 7 } & time $^{7}$ & -0.74 & 0.51 & -1.45 & .15 \\
\cline { 2 - 7 } & time $^{9}$ & 0.69 & 0.50 & 1.38 & .17 \\
\hline \multirow{3}{*}{ Text-only feedback } & time $^{3}$ & 1.05 & 0.52 & 2.01 & $.04^{*}$ \\
\cline { 2 - 7 } & time $^{7}$ & 1.13 & 0.49 & 2.31 & $.02^{*}$ \\
\cline { 2 - 7 } & time $^{9}$ & -1.77 & 0.50 & -3.55 & $.00^{* *}$ \\
\hline
\end{tabular}

$* p<.05$

$* * p<.01$
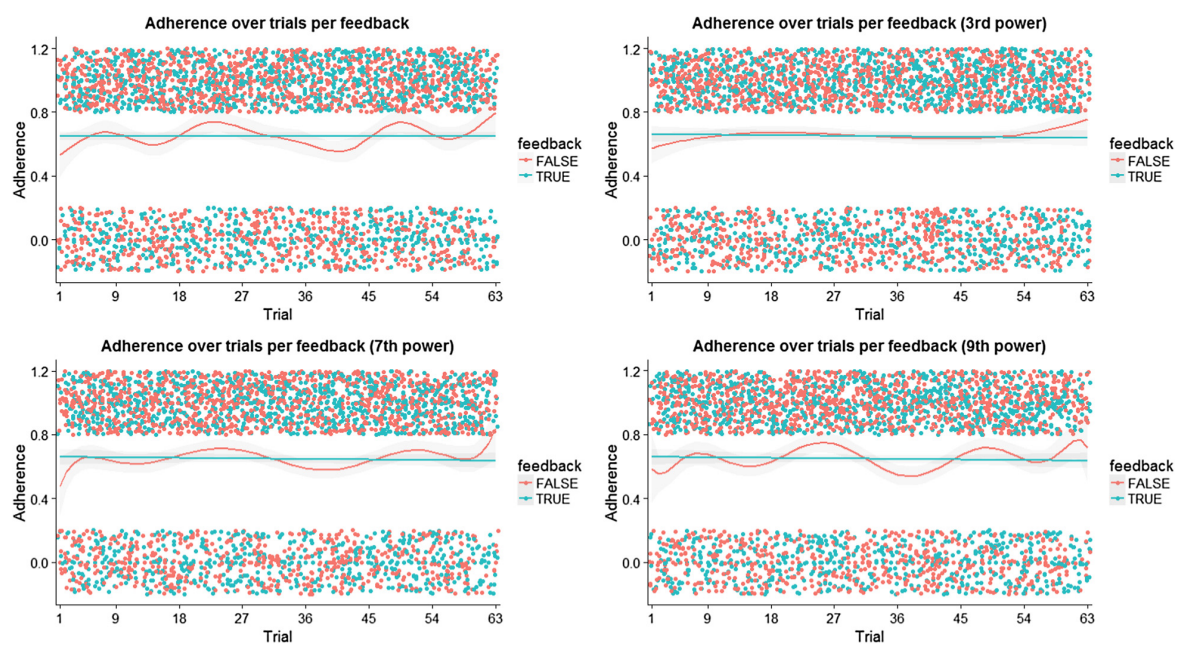

Fig. 4. Visualizations of the overall model (top left), and the three significant polynomial effects, using a smoothed conditional means function 


\section{Discussion}

\subsection{Principal Results}

Our visual feedback did not manage to increase EMA adherence, but rather kept it stable over time. A cautious interpretation of the results is that personalized visual feedback helped to maintain adherence to EMA in a more predictive manner than when text-only feedback was provided. Notably, as is visualized in Fig. 4, there was no positive or negative linear trend for either condition. This is a surprising result given the assumption that adherence would decrease over time.

\subsection{Implications}

From a practical point of view, our results are interesting in the sense that from a researcher perspective, a constant flow of information, as provided by participants in the personalized visual feedback condition, may be preferable to the fluctuations observed in the text-only group. Mood, for example, changes relatively slowly over the course of more than one day. From a theoretical point of view, the results are harder to interpret, since the text-only group was at times more, and at times less adherent than the visual feedback group. Since contextual factors (e.g. having time for EMA responses) were controlled for by our randomization, the difference, i.e., fluctuation versus stabilization, can most likely be explained by an effect on participants' motivation. This would require further investigation; a possible future research paradigm to investigate the effect may include a qualitative component, e.g. by asking participants for their reasons to respond or not respond to EMA requests. It could also be interesting to look more closely at the moods that were reported per day as, for example, participants may be less inclined to answer requests when they are having a bad day compared to a good one.

Although personalized visual feedback seemed to have a stabilizing effect on adherence, it failed to increase it. It could be that personalized visual feedback does not matter that much, but it is also possible that the type of visual feedback we presented is not very effective, or cancelled out any positive effects. With regard to mood mirroring, for example, reflecting negative moods may actually amplify them [24], and thereby decrease user motivation. This was also argued following a study where a virtual agent mirrored users' emotional states to motivate them to play a game [25], in which no significant effects on user motivation were found. Within the context of eMental Health interventions related to mood and our current paradigm, one interesting option for future research could be to investigate the effect of mirroring moods that have a negative valence with their equivalents on the positive side of the valence dimension. For example, a reported irritated $\operatorname{mood}($ valence $=-1$, arousal $=1$ ) would be mirrored by an excited mood (valence $=1$, arousal $=1$ ), which could alleviate the potential drawbacks of reflecting negative mood states. 


\subsection{Limitations}

The virtual agent was a very simplistic one, which had to do with the technological limitations of the platform that we used to conduct the study. For example, the input for the 'reasoning engine' (Fig. 2) of the embodied virtual agent was limited to the two questions that were answered in the current trial, and the EMA framework offered no options for more advanced animations. Although the agent was hardly impressive from a technological perspective, it did allow us to conduct research on its relationship to EMA adherence, within the limited degrees of freedom for agent design offered by a typical eMental Health framework. Additionally, we did so in a well-controlled paradigm: intervention with agent vs. intervention without agent.

It could also be questioned whether our specific feedback is the optimal one in this setting. We chose this feedback as it operationalized elements from Persuasive System Design theory (social role and similarity) known to be beneficial to adherence, and for pragmatic reasons related to the technological limitations of the EMA framework. Although it can be argued that similarity often refers more to user characteristics such as age or gender, we considered mood reflection relevant in our case since the task at hand was mood reporting. Some types of feedback that could be equally, if not more, relevant include reminders before EMA requests, targeting feedback only at participants who have been non-adherent for a period of time, or feedback that is specifically designed to uplift participants who report a negative mood.

A last limitation refers to the generalizability of our results. First and foremost, our study was conducted with a convenience sample, whose primary motivation for participation was likely to help out the students by whom they were recruited. Known mental health issues being an exclusion criterion, our study population was most likely quite different from the clinical populations that would typically use eMental Health interventions, and that may instead be motivated by a desire to improve their current situation. Additionally, there is still some debate as to whether EMA can be considered an intervention in itself [26], which means generalizations to our broader context should be made with caution. These limitations are a natural consequence of the exploratory nature of our pilot study, but the methods we used could be applied equally well to contexts with real interventions and patients.

\section{Conclusion}

The study described in this paper was the first in a series of experiments which we hope will contribute to the empirical validation of the clinical effectiveness of embodied virtual agents in an eMental Health context. We aimed to find out whether feedback, operationalized by an embodied virtual agent, could increase adherence to mood rating requests in a three-week smartphone-based EMA study. While we did not find a significant main effect of feedback on adherence, there was a significant feedback * time interaction effect, which became apparent in fluctuations in adherence for the text-only condition, compared to a very consistent pattern in the personalized visual feedback group. To our knowledge, this paper represents one of the first explorative studies that used an embodied virtual agent, in a rigorous randomized and controlled design, to study a clinically relevant 
outcome measure over a prolonged period of time. Given the explorative nature and the relatively small sample size of this study, the stabilizing effect the virtual agent had on adherence has to be interpreted with some caution. Future studies may include a more sophisticated virtual agent, different feedback, a clinical study population, and a context more resembling CBT interventions.

\section{References}

1. Andersson, G., Cuijpers, P., Carlbring, P., Riper, H., Hedman, E.: Guided internet-based vs. face-to-face cognitive behavior therapy for psychiatric and somatic disorders: a systematic review and meta-analysis. World Psychiatry 13, 288-295 (2014)

2. Richards, D., Richardson, T.: Computer-based psychological treatments for depression: a systematic review and meta-analysis. Clin. Psychol. Rev. 32, 329-342 (2012)

3. Schueller, S.M., Tomasino, K.N., Mohr, D.C.: Integrating human support into behavioral intervention technologies: the efficiency model of support. Clin. Psychol. Sci. Pract. 24, 27 45 (2017)

4. Mohr, D.C., Cuijpers, P., Lehman, K.: Supportive accountability: a model for providing human support to enhance adherence to eHealth interventions. J. Med. Internet Res. 13, e30 (2011)

5. Van Ballegooijen, W., Cuijpers, P., Van Straten, A., Karyotaki, E., Andersson, G., Smit, J. H., Riper, H.: Adherence to internet-based and face-to-face cognitive behavioural therapy for depression: a meta-analysis. PLoS ONE 9, e100674 (2014)

6. Donkin, L., Christensen, H., Naismith, S.L., Neal, B., Hickie, I.B., Glozier, N.: A systematic review of the impact of adherence on the effectiveness of e-therapies. J. Med. Internet Res. 13, e52 (2011)

7. Provoost, S., Lau, H.M., Ruwaard, J., Riper, H.: Embodied conversational agents in clinical psychology: a scoping review. J. Med. Internet Res. 19, e151 (2017)

8. Shiffman, S., Stone, A.A., Hufford, M.R.: Ecological momentary assessment. Annu. Rev. Clin. Psychol. 4, 1-32 (2008)

9. Wenze, S.J., Miller, I.W.: Use of ecological momentary assessment in mood disorders research. Clin. Psychol. Rev. 30, 794-804 (2010)

10. Isbister, K., Doyle, P.: The blind men and the elephant revisited. In: Ruttkay, Z., Pelachaud, C. (eds.) From Brows to Trust. HIS, vol. 7, pp. 3-26. Springer, Dordrecht (2004). https://doi. org/10.1007/1-4020-2730-3_1

11. Bickmore, T., Schulman, D., Shaw, G.: DTask and LiteBody: open source, standards-based tools for building web-deployed embodied conversational agents. In: Ruttkay, Z., Kipp, M., Nijholt, A., Vilhjálmsson, H.H. (eds.) IVA 2009. LNCS (LNAI), vol. 5773, pp. 425-431. Springer, Heidelberg (2009). https://doi.org/10.1007/978-3-642-04380-2_46

12. Gratch, J., Hartholt, A.: Virtual humans: a new toolkit for cognitive science research. Proc. Cogn. Sci. Soc. 35, 41-42 (2013)

13. Mumm, J., Mutlu, B.: Designing motivational agents: the role of praise, social comparison, and embodiment in computer feedback. Comput. Hum. Behav. 27, 1643-1650 (2011)

14. Bickmore, T., Schulman, D., Sidner, C.L.: Issues in Designing Agents for Long-Term Behavior Change. In: CHI Workshop on Engagement by Design, pp. 1-5 (2009)

15. movisensXS, Version 0.7.4162 (movisens GmbH, Karlsruhe, Germany). https://xs. movisens.com/

16. Russell, J.A.: A circumplex model of affect. J. Pers. Soc. Psychol. 39, 1161-1178 (1980) 
17. Asselbergs, J., Ruwaard, J., Ejdys, M., Schrader, N., Sijbrandij, M., Riper, H.: Mobile phone-based unobtrusive ecological momentary assessment of day-to-day mood: an explorative study. J. Med. Internet Res. 18, e72 (2016)

18. Desmet, P.M.A., Vastenburg, M.H., Van Bel, D., Romero, N.: Pick-a-mood development and application of a pictorial mood-reporting instrument. In: Proceedings of the 8th International Conference on Design and Emotion, London 2012, Central Saint Martins College, Art and Design, 11-14 September 2012 (2012)

19. Oinas-Kukkonen, H., Harjumaa, M.: Persuasive systems design: key issues, process model, and system features. Commun. Assoc. Inf. Syst. 24, 485-500 (2009)

20. Kelders, M.S., Kok, N.R., Ossebaard, C.H., Van Gemert-Pijnen, E.W.C.J.: Persuasive system design does matter: a systematic review of adherence to web-based interventions. J Med Internet Res. 14, e152 (2014)

21. Broderick, J.E., Schwartz, J.E., Shiffman, S., Hufford, M.R., Stone, A.A.: Signaling does not adequately improve diary compliance. Ann. Behav. Med. 26, 139-148 (2003)

22. Bates, D., Mächler, M., Bolker, B., Walker, S.: Fitting linear mixed-effects models using lme4. J. Stat. Softw. 67, 1-48 (2015)

23. R Core Team: R: A Language and Environment for Statistical Computing (2016). https:// www.r-project.org

24. Pagliari, C., Burton, C., McKinstry, B., Szentatotai, A., David, D., Serrano Blanco, A., Ferrini, L., Albertini, S., Castro, J.C., Estevez, S., Wolters, M.: Psychosocial implications of avatar use in supporting therapy for depression. Stud. Health Technol. Inform. 181, 329-333 (2012)

25. Burleson, W.: Affective learning companions: strategies for empathetic agents with real-time multimodal affective sensing to foster meta-cognitive and meta-affective approaches to learning, motivation, and perseverance (2006). http://hdl.handle.net/1721.1/37404

26. van Ballegooijen, W., Ruwaard, J., Karyotaki, E., Ebert, D.D., Smit, J.H., Riper, H.: Reactivity to smartphone-based ecological momentary assessment of depressive symptoms (MoodMonitor): protocol of a randomised controlled trial. BMC Psychiatry 16, 359 (2016) 